\title{
What is the role of autoimmunity in type 1 diabetes? A clinical perspective
}

\author{
Marc Y. Donath • Christoph Hess • Ed Palmer
}

Received: 30 September 2013 / Accepted: 6 December 2013 / Published online: 4 January 2014

(C) Springer-Verlag Berlin Heidelberg 2014

\begin{abstract}
Despite tremendous research efforts, type 1 diabetes is one of the few remaining autoimmune diseases without any approved immunological treatment. This observation compels us to reconsider the role of autoimmunity in the pathogenesis of this disease. In this commentary, we will review solely human data in an attempt to appreciate, in an unbiased manner, the importance and relevance of the immunological alterations in patients with type 1 diabetes. The aim of this paper is to generate reflection on this topic, rather than a controversy.
\end{abstract}

Keywords Autoimmunity $\cdot$ Beta cells $\cdot$ Clinic $\cdot$

Type 1 diabetes

A recent expert workshop reviewed the histopathological characteristics of insulitis in human type 1 diabetes [1]. It was noted that, in humans, only 150-200 cases have been described. The emerging picture of insulitis (described in the consensus definition of insulitis) is that the fraction of infiltrated islets is very low $(<10 \%)$, and the infiltrated islets

M. Y. Donath $(\bowtie)$

Endocrinology, Diabetes and Metabolism,

University Hospital Basel and University of Basel, Petersgraben 4,

CH-4031 Basel, Switzerland

e-mail: marc.donath@usb.ch

M. Y. Donath $\cdot$ C. Hess $\cdot$ E. Palmer

Department of Biomedicine, University Hospital Basel and

University of Basel, Basel, Switzerland

C. Hess

Medical Outpatient Clinic, University Hospital Basel and University of Basel, Basel, Switzerland

E. Palmer

Laboratory of Transplantation Immunology, University Hospital

Basel and University of Basel, Basel, Switzerland contain very few immune cells ( $\geq 15$ leucocytes/islet is considered as positive for infiltration, which is only twice the number of leucocytes found in control donor pancreases [2]). Moreover, infiltrating leucocytes are often found only in the islet periphery (peri-insulitis). Although islet-autoreactive $\mathrm{CD}^{+} \mathrm{T}$ cells were detected [3], the level of insulitis remains surprisingly mild, even in patients with recent-onset type 1 diabetes and death due to ketoacidosis [4]. This picture is complicated by background infiltration in the exocrine pancreas, partly due to peri-mortem changes, with the highest levels of infiltration observed in young patients after prolonged life support [5]. Even independently of postmortem changes, type 1 diabetes affects the exocrine as well as the endocrine pancreas [6]; this raises the question whether the limited infiltration of leucocytes is specifically targeted to pancreatic beta cells. Moreover, this limited degree of cellular infiltration in human autopsy samples is in striking contrast to NOD mice, which display massive leucocyte infiltration, reflecting the well-documented immune-mediated destruction of the beta cells in this animal model. Interestingly, the cellular infiltrates observed in NOD mouse islets are reminiscent of histopathological findings in organs affected by 'classical' human autoimmune diseases, such as rheumatoid arthritis, psoriasis, arteritis and colitis (see e.g. Lahmar et al [7]). Even strictly antibody-mediated autoimmunity, such as the antiglomerular basement syndrome, is accompanied by a relevant influx of inflammatory leucocytes [8]. Therefore, based on the available human histological findings in patients with type 1 diabetes, it is difficult to relate such a mild immune cell infiltration to the devastating consequences on insulin secretion observed in the clinic.

Even more puzzling is the outcome of clinical studies in patients with type 1 diabetes: although encouraging, they need to be put into context. The ciclosporin trials are often quoted as proof of concept studies, demonstrating the autoimmune nature of the disease $[9,10]$; however, several limitations 
question such a definitive conclusion and, indeed, the absolute number of responders was low. In one study, only $25.4 \%$ of the ciclosporin group vs $18.6 \%$ of the placebo group were in complete remission at 6 months, which is not statistically significant; only sub-analyses showed statistically significant differences [10]. A follow-up study did achieve statistical significance with a higher rate of remission, but in absolute terms the efficacy remained rather modest (33\% remission with ciclosporin compared with $22 \%$ with placebo) [9]. Of note, in both studies ciclosporin treatment did not translate into improvement in $\mathrm{HbA}_{1 \mathrm{c}}$ levels. Furthermore, the appearance of hypertrichosis and gingival hyperplasia (both typical side effects of ciclosporin treatment) indirectly unblinded the study. Other untoward effects such as abdominal discomfort, nausea, vomiting, diarrhoea and infections, or off-target effects, may also explain, at least in part, the metabolic effects of ciclosporin on diabetes - independent of its immunosuppressive effects. The lack of a temporal correlation between changes in islet cell antibodies and changes in C-peptide levels are also in line with this supposition [11]. Indeed, this observation has given rise to the hypothesis that the generation of autoantibodies in type 1 diabetes may be a secondary event triggered by a primary islet-destructive process. Follow-up studies using other immune modulatory treatments, including cytotoxic T-lymphocyte antigen 4 (CTLA4)-immunoglobulin fusion protein [12], anti-CD20 antibody [13], anti-CD3 antibody [14-16], DiaPep277 [17], TNF- $\alpha$ receptor fusion protein [18] and anti-IL-1 $\beta$ antibody or IL-1 receptor antagonist [19], showed mild initial benefit, or no benefit at all. Of note, in cases where clinical benefits were observed, the typical pattern was an initial improvement in insulin production, followed by a decline which paralleled that observed in untreated patients. Taken together, these data suggest that deleterious effects on beta cell function attributable to the immune system are small, and that a separate pathogenic mechanism is the main driving force of beta cell loss. The limited and partial improvement observed in patients with type 1 diabetes contrasts with the profound benefit observed with similar treatments in other autoimmune diseases; see for example that TNF- $\alpha$ antagonism in Crohn's disease induces a response in $80 \%$ of cases compared with $17 \%$ in placebo-treated patients [20]. Of note, the lack of efficacy of immunomodulatory therapy in patients with type 1 diabetes cannot be attributed to the timing of therapeutic intervention, because most studies were performed within 3 months after the onset of hyperglycaemia, a time point when there is still sufficient beta cell mass present that could potentially be rescued [21]. Indeed, most patients experience a spontaneous remission for weeks or months following the initial onset of hyperglycaemia (referred to as the 'honeymoon'), which is possibly caused by a reversal of glucotoxicity. Therefore, the time and duration of the immune intervention (several months in most trials) should have been sufficient to achieve similar effects to those occurring naturally during the honeymoon. However, our speculation should in no way discourage ongoing or planned clinical studies evaluating the abovementioned or other immune modulating drugs: future studies, using other designs or combinations of drugs may prove us wrong.

In some patients, type 1 diabetes is associated with other autoimmune disease. This association is often put forward as an argument for an autoimmune aetiology of type 1 diabetes. Nevertheless, this association is not a general trait of type 1 diabetes, but the situation is rather that a small fraction of type 1 diabetes cases are associated with very specific diseases, mainly Hashimoto's thyroiditis, adrenal insufficiency, vitiligo and coeliac disease. To explain such an association by a defect in the adaptive immune system, one might expect some mechanism that would target the immune system specifically to these different tissues. Yet at molecular level, there is no obvious similarity between the affected tissues, for example the steroid-producing adrenal cells and the peptide hormonereleasing beta cells. However, it is noteworthy that all the above-mentioned diseases affect active, secretory cells. Therefore, it is conceivable that the primary shared defect is a secretory dysfunction (possibly inherited) leading to cell destruction. As a secondary event, excessive auto-antigen presentation will occur and peripheral tolerance may eventually be lost. Indeed, a pathological activation of the immune system is observed in most patients with type 1 diabetes, as reflected by the presence of auto-antibodies and $\mathrm{T}$ cells targeting beta cell-derived antigens. Nevertheless, such activation is also observed in many diseases involving cell death, including type 2 diabetes [22]. Thus, the question is whether these immunological phenomena are the main drivers, or merely epiphenomena, in the pathogenesis of type 1 diabetes.

The strong linkage of type 1 diabetes with HLA genotype favours an autoimmune aetiology [23]. However this cannot be considered as a final proof, since this chromosomal region encodes hundreds of genes, which may also be involved in beta cell demise by other mechanisms. Furthermore, type 1 diabetes does not segregate with any clear mode of inheritance. Along the same lines, the interesting observation that recurrence of disease has been prevented by immunosuppression in a few cases of recipients of monozygotic twin semipancreatic grafts [24] may point to an autoimmune aetiology, but the incomplete description of the immunological reaction and the complex setting of organ transplantation do not rule out other causes.

Thus we are left to ponder the true nature of the disease. Adding to the complexity of this issue is the heterogeneity between patients and the histological heterogeneity within any given pancreas [1], most probably reflecting distinct subtypes of diabetes. It is conceivable that true autoimmunity occurs in a subtype of patients; however, both in the histopathology studies and in the clinical trials discussed above, cases with 
marked leucocyte infiltration or with remission following immunosuppressive treatment are rare. Therefore, we believe that an open-minded effort should be made to understand the primary aetiology of type 1 diabetes, instead of focusing on downstream events and pathways that may or may not be relevant. The Network for Pancreatic Organ Donors with Diabetes initiative, supported by the JDRF, has led to the above-mentioned review of human pancreas histology [1]; this is a step in right direction.

Duality of interest The authors declare that there is no duality of interest associated with this manuscript

Contribution statement All authors were responsible for the conception and design of the manuscript, drafting the article and revising it critically for important intellectual content. All authors approved the version to be published.

\section{References}

1. Campbell-Thompson ML, Atkinson MA, Butler AE et al (2013) The diagnosis of insulitis in human type 1 diabetes. Diabetologia 56: 2541-2543

2. In't Veld P, Lievens D, de Grijse J et al (2007) Screening for insulitis in adult autoantibody-positive organ donors. Diabetes 56:2400-2404

3. Coppieters KT, Dotta F, Amirian N et al (2012) Demonstration of islet-autoreactive CD8 T cells in insulitic lesions from recent onset and long-term type 1 diabetes patients. J Exp Med 209:51-60

4. Willcox A, Richardson SJ, Bone AJ, Foulis AK, Morgan NG (2009) Analysis of islet inflammation in human type 1 diabetes. Clin Exp Immunol 155:173-181

5. In't Veld P, de Munck N, van Belle K et al (2010) Beta-cell replication is increased in donor organs from young patients after prolonged life support. Diabetes 59:1702-1708

6. Skog O, Korsgren S, Melhus A, Korsgren O (2013) Revisiting the notion of type 1 diabetes being a T cell-mediated autoimmune disease. Curr Opin Endocrinol Diabetes Obes 20:118-123

7. Lahmar A, Mzabi-Regaya S, Boubaker S, Kchir N, Hendaoui L (2012) Systemic vasculitis: anatomy and histopathology. In: Hendaoui L, Stanson AW, Habib Bouhaouala M, Joffre F (eds) Systemic vasculitis. Springer, Heidelberg, pp 15-23

8. Cui Z, Zhao MH (2011) Advances in human antiglomerular basement membrane disease. Nat Rev Nephrol 7:697-705

9. The Canadian-European Randomized Control Trial Group (1988) Cyclosporin-induced remission of IDDM after early intervention.
Association of $1 \mathrm{yr}$ of cyclosporin treatment with enhanced insulin secretion. Diabetes 37:1574-1582

10. Feutren G, Papoz L, Assan R et al (1986) Cyclosporin increases the rate and length of remissions in insulin-dependent diabetes of recent onset. Results of a multicentre double-blind trial. Lancet 2: $119-124$

11. Mandrup-Poulsen T, Nerup J, Stiller CR et al (1985) Disappearance and reappearance of islet cell cytoplasmic antibodies in cyclosporintreated insulin-dependent diabetics. Lancet 1:599-602

12. Orban T, Bundy B, Becker DJ et al (2011) Co-stimulation modulation with abatacept in patients with recent-onset type 1 diabetes: a randomised, double-blind, placebo-controlled trial. Lancet 378: 412-419

13. Pescovitz MD, Greenbaum CJ, Krause-Steinrauf H et al (2009) Rituximab, B-lymphocyte depletion, and preservation of beta-cell function. N Engl J Med 361:2143-2152

14. Herold KC, Hagopian W, Auger JA et al (2002) Anti-CD3 monoclonal antibody in new-onset type 1 diabetes mellitus. $\mathrm{N}$ Engl J Med 346:1692-1698

15. Keymeulen B, Vandemeulebroucke E, Ziegler AG et al (2005) Insulin needs after $\mathrm{CD} 3$-antibody therapy in new-onset type 1 diabetes. N Engl J Med 352:2598-2608

16. Sherry N, Hagopian W, Ludvigsson J et al (2011) Teplizumab for treatment of type 1 diabetes (Protege study): 1-year results from a randomised, placebo-controlled trial. Lancet 378:487-497

17. Huurman VA, Decochez K, Mathieu C, Cohen IR, Roep BO (2007) Therapy with the hsp60 peptide DiaPep277 in C-peptide positive type 1 diabetes patients. Diabetes Metab Res Rev 23:269-275

18. Mastrandrea L, Yu J, Behrens T et al (2009) Etanercept treatment in children with new-onset type 1 diabetes: pilot randomized, placebo-controlled, double-blind study. Diabetes Care 32: $1244-1249$

19. Moran A, Bundy B, Becker DJ et al (2013) Interleukin-1 antagonism in type 1 diabetes of recent onset: two multicentre, randomised, double-blind, placebo-controlled trials. Lancet 381:1905-1915

20. Targan SR, Hanauer SB, van Deventer SJ et al (1997) A short-term study of chimeric monoclonal antibody cA2 to tumor necrosis factor alpha for Crohn's disease. Crohn's Disease cA2 Study Group. N Engl J Med 337:1029-1035

21. Greenbaum CJ, Beam CA, Boulware D et al (2012) Fall in C-peptide during first 2 years from diagnosis: evidence of at least two distinct phases from composite Type 1 Diabetes TrialNet data. Diabetes 61: 2066-2073

22. Brooks-Worrell B, Narla R, Palmer JP (2012) Biomarkers and immune-modulating therapies for type 2 diabetes. Trends Immunol 33:546-553

23. Concannon P, Rich SS, Nepom GT (2009) Genetics of type 1A diabetes. N Engl J Med 360:1646-1654

24. Sutherland DE, Goetz FC, Sibley RK (1989) Recurrence of disease in pancreas transplants. Diabetes 38(Suppl 1):85-87 